\title{
Exploring partial $\mu-\tau$ reflection symmetry at DUNE and Hyper-Kamiokande
}

\author{
Kaustav Chakraborty, ${ }^{1,2, *}$ K. N. Deepthi, ${ }^{1, \dagger}$ Srubabati Goswami, ${ }^{1, \$}$ \\ Anjan S. Joshipura, ${ }^{1,8}$ and Newton Nath ${ }^{3,4, \pi}$ \\ ${ }^{1}$ Theoretical Physics Division, Physical Research Laboratory, \\ Ahmedabad 380009, India \\ ${ }^{2}$ Discipline of Physics, Indian Institute of Technology, Gandhinagar 382355, India \\ ${ }^{3}$ Institute of High Energy Physics, Chinese Academy of Sciences, \\ Beijing 100049, China \\ ${ }^{4}$ School of Physical Sciences, University of Chinese Academy of Sciences, \\ Beijing 100049, China
}

(Received 12 April 2018; revised manuscript received 11 October 2018; published 31 October 2018)

We study the origin, consequences, and testability of a hypothesis of "partial $\mu$ - $\tau$ " reflection symmetry. This symmetry predicts $\left|U_{\mu i}\right|=\left|U_{\tau i}\right|(i=1,2,3)$ for a single column of the leptonic mixing matrix $U$. Depending on whether this symmetry holds for the first or second column of $U$, different correlations between $\theta_{23}$ and $\delta_{C P}$ can be obtained. This symmetry can be obtained using discrete flavor symmetries. In particular, all of the subgroups of SU(3) with three-dimensional irreducible representation, which are classified as class $\mathrm{C}$ or $\mathrm{D}$, can lead to partial $\mu-\tau$ reflection symmetry. We show how the predictions of this symmetry compares with the allowed area in the $\sin ^{2} \theta_{23}-\delta_{C P}$ plane as obtained from the global analysis of neutrino oscillation data. Furthermore, we study the possibility of testing these symmetries at the proposed DUNE and Hyper-Kamiokande (HK) experiments (T2HK, T2HKK), by incorporating the correlations between $\theta_{23}$ and $\delta_{C P}$ that are predicted by the symmetries. We find that when the simulated data of DUNE and $\mathrm{HK}$ are fitted with the symmetry predictions, the $\theta_{23}-\delta_{C P}$ parameter space gets largely restricted near the charge parity conserving values of $\delta_{C P}$. Finally, we illustrate the capability of these experiments to distinguish between the two cases leading to partial $\mu-\tau$ symmetry, namely $\left|U_{\mu 1}\right|=\left|U_{\tau 1}\right|$ and $\left|U_{\mu 2}\right|=\left|U_{\tau 2}\right|$.

DOI: 10.1103/PhysRevD.98.075031

\section{INTRODUCTION}

Considerable theoretical and experimental efforts are being devoted towards predicting and determining the unknowns of the leptonic sectors, namely the charge parity $(C P)$ violating phase, octant of the atmospheric mixing angle $\theta_{23}$ [i.e., $\theta_{23}<45^{\circ}$, named as lower octant (LO) or $\theta_{23}>45^{\circ}$ named as upper octant (HO)], and the neutrino mass hierarchy [i.e., the sign of $\Delta m_{31}^{2}, \Delta m_{31}^{2}>0$ known as normal hierarchy $(\mathrm{NH})$ and $\Delta m_{31}^{2}<0$ known as inverted hierarchy $(\mathrm{IH})]$. Symmetry based approaches have

\footnotetext{
*kaustav@prl.res.in

†deepthi@prl.res.in

\$sruba@prl.res.in

$\S$ anjan@prl.res.in

"newton@ihep.ac.cn
}

Published by the American Physical Society under the terms of the Creative Commons Attribution 4.0 International license. Further distribution of this work must maintain attribution to the author(s) and the published article's title, journal citation, and DOI. Funded by SCOAP. been quite successful in predicting the interrelations among these quantities and the structure of the leptonic mixing matrix as discussed in Refs. [1-5] and the references therein. General approaches along this line assume some individual residual symmetries of the leptonic mass matrices that could arise from the breaking of some of the bigger symmetry of the leptonic interactions. One such symmetry, called $\mu-\tau$ reflection symmetry, originally discussed by Harrison and Scott in Ref. [6], leads to very successful predictions of mixing angles that are close to the present experimental knowledge. This symmetry may be stated as an equality of moduli of the leptonic mixing matrix $U$ :

$$
\left|U_{\mu i}\right|=\left|U_{\tau i}\right|
$$

for all of the columns $i=1,2,3$. Both the origin and consequences of this relation have been discussed in [7-22].

Using the standard Particle Data Group (PDG) [23] parametrization of the matrix $U$ 


$$
U=U\left(\theta_{23}\right) U\left(\theta_{13}, \delta_{C P}\right) U\left(\theta_{12}\right)=\left[\begin{array}{ccc}
c_{12} c_{13} & s_{12} c_{13} & s_{13} e^{-i \delta_{C P}} \\
-s_{12} c_{23}-c_{12} s_{23} s_{13} e^{i \delta_{C P}} & c_{12} c_{23}-s_{12} s_{23} s_{13} e^{i \delta_{C P}} & s_{23} c_{13} \\
s_{12} s_{23}-c_{12} c_{23} s_{13} e^{i \delta_{C P}} & -c_{12} s_{23}-s_{12} c_{23} s_{13} e^{i \delta_{C P}} & c_{23} c_{13}
\end{array}\right]
$$

one finds two well-known predictions

$$
\theta_{23}=\frac{\pi}{4}, \quad s_{13} \cos \delta_{C P}=0
$$

Equation (3) suggests a maximal $\theta_{23}$, which is allowed within $1 \sigma$ by the global fits to neutrino observables [24-26]. Additionally, it allows a nonzero $\theta_{13}$, unlike the simple $\mu-\tau$ symmetry that predicts vanishing $\theta_{13}$ [27-32], see recent review [33] and references therein. Here, for $\theta_{13} \neq 0$, one gets $\delta_{C P}= \pm \frac{\pi}{2}$ using Eq. (3). Both of these predictions are in accord with the global fit of all neutrino data. However, a sizeable range is still allowed at $3 \sigma$. Note that, the best fit value of $\theta_{23}$ in the global fit deviates from the maximal value for either mass hierarchy. Such deviations can be regarded as a signal for the departure from the $\mu-\tau$ reflection symmetry. A theoretically well-motivated possibility is to assume a "partial $\mu-\tau$ " reflection symmetry [34] and assume that Eq. (1) holds only for a single column" of $U$. Assuming that it holds for the third column, one gets the maximal $\theta_{23}$ and $\delta_{C P}$ remains unrestricted. These correlations are found from Eq. (2) in respective cases $i=1$ and $i=2$ to be

$$
\begin{aligned}
\cos \delta_{C P}= & \frac{\left(c_{23}^{2}-s_{23}^{2}\right)\left(c_{12}^{2} s_{13}^{2}-s_{12}^{2}\right)}{4 c_{12} s_{12} c_{23} s_{23} s_{13}}, \\
& \left(\left|U_{\mu 1}\right|=\left|U_{\tau 1}\right|\right), \quad C_{1}, \\
\cos \delta_{C P}= & \frac{\left(c_{23}^{2}-s_{23}^{2}\right)\left(c_{12}^{2}-s_{12}^{2} s_{13}^{2}\right)}{4 c_{12} s_{12} c_{23} s_{23} s_{13}}, \\
& \left(\left|U_{\mu 2}\right|=\left|U_{\tau 2}\right|\right), \quad C_{2} .
\end{aligned}
$$

These equations correlate the sign of $\cos \delta_{C P}$ to the octant of $\theta_{23} . \theta_{23}$ in the first (second) octant leads to a negative (positive) value of $\cos \delta_{C P}$ in the case of Eq. (4). It exactly predicts the opposite behavior for Eq. (5). The exact quadrant of $\delta$ is still not fixed by these equations, but it can also be determined from symmetry considerations [20]. These correlations were also obtained in $[35,36]$ in the context of $Z_{2}$ and $\overline{Z_{2}}$ symmetries. ${ }^{2}$ Henceforth, we refer to these correlations as $C_{1}$ and $C_{2}$, respectively. The above equations also indirectly lead to information on the neutrino mass hierarchy since the best fit values of $\theta_{23}$ lie in the first

\footnotetext{
${ }^{1}$ If it holds for any two columns, then by unitarity, it holds for the third as well.

${ }^{2}$ See the review article [37] for references on other similar sum rules and their testability.
}

(second) octant in the case of the normal (inverted) hierarchy, according to the latest global fits reported in [24-26]. Thus, precise verification of the above equations is of considerable importance, and the long baseline experiments can provide a way for such study. A similar study has been performed in the context of the $\mathrm{NO} \nu \mathrm{A}$ and $\mathrm{T} 2 \mathrm{~K}$ experiments in [38-40].

In this paper, we consider the testability of these relations at the forthcoming long baseline experiments of the Deep Under-ground Neutrino Experiment (DUNE) and HyperKamiokande (HK). These potential high-statistics experiments will overcome the parameter degeneracies faced by the current experiments and lead us in to an era of precision measurements of the oscillation parameters [41-50]. Because of this, these experiments are ideal to test the parameter correlations like the ones given in the Eqs. (4), (5). In the following, we obtain the allowed parameter range in the $\delta_{C P}-\sin ^{2} \theta_{23}$ plane by fitting the symmetry relations embodied in Eqs. (4), (5) to the simulated DUNE and HK data. We also discuss whether the correlations $C_{1}$ [Eq. (4)] and $C_{2}$ [Eq. (5)] can be distinguished at DUNE and HK. Recent studies on testing various models from future experiments can be found, for instance, in [51-59].

We begin by first discussing the origin of partial $\mu-\tau$ reflection symmetry. After which, in Sec. II, we elaborate on the robustness of the resulting predictions in a large class of models based on flavor symmetry. We give a brief overview of the experiments and simulation details in Sec. III. In Sec. IV, we perform a phenomenological analysis of the testability of the above symmetries in the DUNE and HK data. We use the extra correlations predicted by the symmetry in fitting the simulated data of these experiments, and we obtain the allowed areas in the $\delta_{C P}-\sin ^{2} \theta_{23}$ plane. In subsection IV B, we discuss the possibility of differentiating between the two symmetries $-C_{1}$ and $C_{2}$. We draw our conclusions in Sec. V.

\section{PARTIAL $\mu$ - $\tau$ REFLECTION SYMMETRY AND DISCRETE FLAVOR SYMMETRIES}

Here, we briefly review the general approach based on flavor symmetry to emphasize that partial $\mu-\tau$ reflection symmetry is a generic prediction of almost all such schemes, barring a few exceptions. Basic approaches assume groups $G_{\nu}$ and $G_{l}$ as the residual symmetries of the neutrino mass matrix $M_{\nu}$ and the charged lepton mass matrix $M_{l} M_{l}^{\dagger}$, respectively. Both of these groups are assumed to arise from the breaking of some unitary discrete group $G_{f}$. The $U_{\text {PMNS }}$ matrix $U$ gets fixed up to the neutrino Majorana phases if 

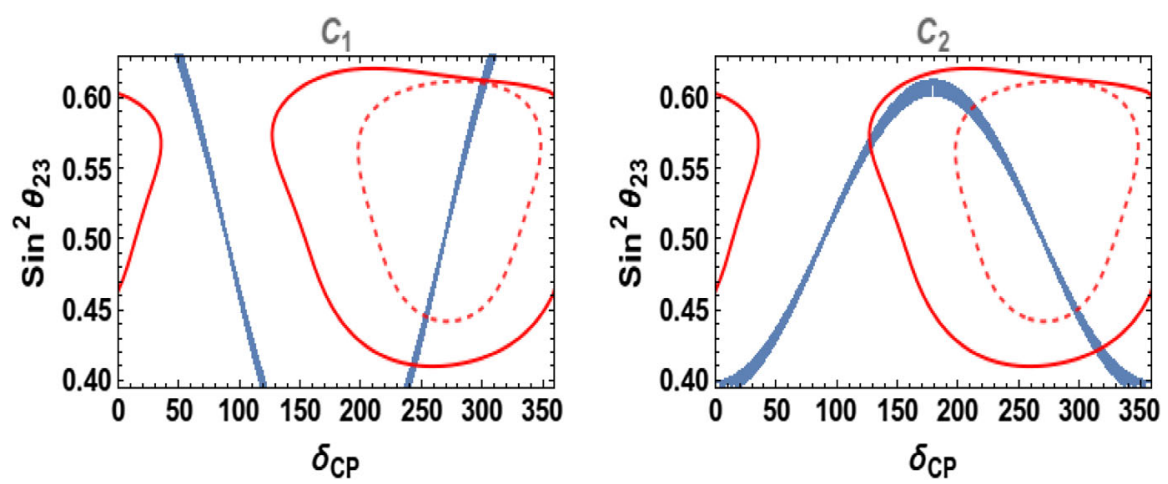

FIG. 1. The thick blue lines show the correlation plots in the $\sin ^{2} \theta_{23}-\delta_{C P}$ plane as predicted by the symmetry relations. The left (right) panel correspond to Eq. (4) [Eq. (5)]. The solid(dashed) red curves represent the $3 \sigma$ allowed parameter space as obtained by the global analysis of data by the Nu-fit Collaboration $[26,63]$ considering the hierarchy to be $\mathrm{NH}(\mathrm{IH})$, respectively.

it is further assumed that $G_{\nu}=Z_{2} \times Z_{2}$ and $G_{l}=Z_{n}, n \geq 3$. In addition, if we demand that all of the predicted mixing angles are nonzero, then the following unique form is predicted for almost all the discrete groups $G_{f}[60,61]$,

$$
\begin{aligned}
U & \equiv U_{\text {gen }}\left(\theta_{n}\right) \\
& =\frac{1}{\sqrt{3}}\left(\begin{array}{ccc}
\sqrt{2} \cos \theta_{n} & 1 & \sqrt{2} \sin \theta_{n} \\
\sqrt{2} \cos \left(\theta_{n}-\frac{2 \pi}{3}\right) & 1 & \sqrt{2} \sin \left(\theta_{n}-\frac{2 \pi}{3}\right) \\
\cos \left(\theta_{n}-\frac{4 \pi}{3}\right) & 1 & \sqrt{2} \sin \left(\theta_{n}-\frac{4 \pi}{3}\right)
\end{array}\right),
\end{aligned}
$$

where $\theta_{n} \equiv \frac{\pi a}{n}$ is a discrete angle with $a=0,1,2 \ldots \frac{n}{2}$. Here, we have not shown the unphysical phases that can be absorbed in defining charged lepton fields and unpredicted Majorana phases. All of the discrete subgroups of SU(3) with three dimensional irreducible representation are classified as class $C$ or $D$ and five exceptional groups [62]. Equation (6) follows in all of the type $D$ groups taken as $G_{f}$. Type $C$ groups lead instead to democratic mixing, which shows full $\mu-\tau$ reflection symmetry but predicts a large reactor angle. Equation (6) arises even if $G_{f}$ is chosen as a discrete subgroup of $U(3)$, having the same textures as class $D$ groups [61].

Equation (6) displays partial $\mu-\tau$ reflection symmetry for the second column for all the values of $\theta_{n} \neq 0, \frac{\pi}{2}$. In the latter case, one gets total $\mu$ - $\tau$ reflection symmetry, but at the same time, one of the mixing angles is predicted to be zero and one would need to break the assumed residual symmetries to get the correct mixing angles. More importantly, Eq. (6) essentially being a real matrix also predicts a trivial Dirac $C P$ phase $\delta_{C P}=0$ or $\pi$. Equation (5) in this case implies a correlation among angles. A nonzero $C P$ phase and partial $\mu-\tau$ symmetry in other columns can arise in an alternative but less predictive approach, in which the residual symmetry of the neutrino mass matrix is taken as $Z_{2}$ instead of $Z_{2} \times Z_{2}$. In this case, one can obtain the following mixing matrix $U$ with a proper choice of residual symmetries

$$
U=U_{\text {gen }}(0) U_{i j},
$$

where $U_{i j}$ denotes a unitary rotation either in the $i j$ th plane corresponding to partial symmetry in the $k$ th $(i \neq j \neq k)$ column. Examples of the required residual symmetries are discussed in [1-5], and a minimal example of this occurs with $G_{f}=S_{4}$.

The partial $\mu$ - $\tau$ symmetries obtained this way also lead to additional restrictions

$$
c_{12}^{2} c_{13}^{2}=\frac{2}{3}
$$

and

$$
s_{12}^{2} c_{13}^{2}=\frac{1}{3},
$$

where Eqs. (8) and (9) follow from the partial symmetries of the first and second columns, respectively. These predictions arise here from the requirement that $G_{\nu}$ and $G_{l}$ are embedded in the Discrete Subgroup of SU(3) and need not arise in a more general approach. It is then possible to obtain specific symmetries $[35,36]$ in which the solar angle is a function of a continuous parameter.

Figure 1, shows the correlation plots (thick blue lines) between $\sin ^{2} \theta_{23}$ and $\delta_{C P}$ as given by Eqs. (4), (5). Here, the red solid(dashed) contours represent the $3 \sigma$ allowed region for $\mathrm{NH}(\mathrm{IH})$ as obtained from the global-fit data by the $\mathrm{Nu}-$ fit Collaboration [63]. Equations (4), (5) give two values of the $C P$ phase (namely, $\delta_{C P}$ and $360^{\circ}-\delta_{C P}$ ) for each value of $\theta_{23}$ except for $\delta_{C P} \equiv 180^{\circ}$. The width of the blue lines is due to the uncertainty of the angles $\theta_{12}$ and $\theta_{13}$, subject to the conditions given in Eqs. (8) and (9), corresponding to Eqs. (4) and (5), respectively. The correlation between $\sin ^{2} \theta_{23}$ and $\delta_{C P}$ is opposite in the class of symmetries that give Eq. (4), vis-à-vis those that give Eq. (5). The parameters, $\sin ^{2} \theta_{23}$ and $\delta_{C P}$, are correlated between $0^{\circ}-180^{\circ}$ and are 
anticorrelated between $180^{\circ}-360^{\circ}$ for Eq. (4). The opposite is true for Eq. (5). We also notice here that Eq. (5) rules out regions around $C P$ conserving (i.e., $0^{\circ}, 180^{\circ}, 360^{\circ}$ ) values. Additionally, we observe that, at $3 \sigma$, some of the allowed regions of $\sin ^{2} \theta_{23}$ and $\delta_{C P}$, as predicted by the symmetries, are disfavored by the current global-fit data. From the globalfit data, we observe that the region $39^{\circ}<\delta_{C P}<125^{\circ}$ is completely ruled out at $3 \sigma$ for the $\mathrm{NH}$ and the region $\delta_{C P}<195^{\circ}$ for the IH. The symmetry predictions can further constrain the values of $\delta_{C P}$ presently allowed by the global data.

In the next section, we study how far the allowed areas in the $\delta_{C P}-\sin ^{2} \theta_{23}$ plane can be restricted if the simulated experimental data confronts the symmetry predictions.

\section{EXPERIMENTAL SPECIFICATIONS}

In this paper, we have simulated all of the experiments using the GLOBES package $[64,65]$ along with the required auxiliary files $[66,67]$. We have considered the experimental setup and the detector performance of DUNE and HK in accordance with Refs. [68] and [69], respectively.

(i) Deep Underground Neutrino Experiment (DUNE): The DUNE is a Fermilab based next generation long baseline superbeam experiment. This experiment will utilize the upcoming leading edge facilitythe Long Baseline Neutrino Facility (LBNF) which will provide a high intensity neutrino beam and the infrastructure required for DUNE. In this experiment, the muon-neutrino beam from Fermilab will travel a baseline of $1300 \mathrm{~km}$ before it gets detected at the far detector, situated at the Sanford Underground Research Facility (SURF) in Lead, South Dakota. The proposed far detector for DUNE is a liquid argon time-projection chamber (LARTPC) detector with a volume of $40 \mathrm{kT}$. The beam power will be initially $1.2 \mathrm{MW}$, and later, it will be increased to $2.3 \mathrm{MW}$ [70]. In our simulation, we consider the neutrino flux [71] corresponding to 1.2 MW beam power, which gives $1 \times 10^{21}$ protons on target (POT) per year. This corresponds to a proton energy of $120 \mathrm{GeV}$. We also consider a total run time of $(5 \nu+5 \bar{\nu})$ years as proposed by the experiment.

(ii) Hyper-Kamiokande Experiment: The HyperKamiokande experiment [69] is a Japanese based long baseline experiment that will use the Japan Proton Accelerator Research Complex (J-PARC) neutrino beam facility. The primary goal of the HK experiment is to determine $C P$ violation. However, it is also capable of observing nucleon decay, atmospheric neutrinos, and neutrinos of astronomical origin. Recently, the collaboration has proposed two alternatives for the location of the far detector. The first one is Tokai-to-HyperKamiokande (T2HK), which plans on constructing two water-cherenkov detectors (cylindrical tanks) of fiducial volume $187 \mathrm{kt}$ at $295 \mathrm{~km}$ in Kamioka. Alternatively, T2HKK proposes to have one tank of $187 \mathrm{kt}$ at $295 \mathrm{~km}$ in Kamioka and the other $187 \mathrm{kt}$ tank at $1100 \mathrm{~km}$ in Korea [69]. In our simulations, we have considered the off axis angle (OAA) for this detector in Korea as $1.5^{\circ}$, a proposed run time ratio to be $1: 3$ in neutrino and antineutrino modes (total run time 10 years), and the proton beam power of $1.3 \mathrm{MW}$, giving a total of $27 \times 10^{21}$ protons on target (POT).

\section{PHENOMENOLOGICAL ANALYSIS}

In this section, we perform a phenomenological analysis exploring the possibility of probing the correlations $C_{1}$ and $C_{2}$ at DUNE, T2HK, and T2HKK. This is discussed in terms of correlation plots in the $\sin ^{2} \theta_{23}-\delta_{C P}$ plane. We also discuss the possibility of distinguishing between the two models at these experiments.

We perform a $\chi^{2}$ test with $\chi^{2}$ defined as,

$$
\chi_{\text {tot }}^{2}=\min _{\xi, \omega}\left\{\chi_{\text {stat }}^{2}(\omega, \xi)+\chi_{\text {pull }}^{2}(\xi)+\chi_{\text {prior }}^{2}\right\} .
$$

$\chi_{\text {stat }}^{2}$ is the statistical $\chi^{2}$, whereas $\chi_{\text {pull }}^{2}$ signifies the systematic uncertainties, which are included using the method of pulls with $\xi$ denoting the pull variable [72-74]. Here, $\omega$ represents the oscillation parameters: $\left\{\sin ^{2} \theta_{23}, \sin ^{2} \theta_{12}\right.$, $\left.\delta_{C P}, \Delta m_{21}^{2}, \Delta m_{31}^{2}\right\} \cdot \chi_{\text {prior }}^{2}$ captures the knowledge of the oscillation parameters from other experiments and is defined as,

$$
\chi_{\text {prior }}^{2}(p)=\frac{\left(p_{0}-p\right)^{2}}{\sigma_{0}^{2}},
$$

$p$ denotes the parameter for which the prior is added, and $p_{0}$ and $\sigma_{0}$ correspond to its best fit value and $1 \sigma$ error, respectively. In our analysis we have considered the effect of the prior on the parameters $\theta_{13}$ and $\theta_{12}$. We assume a Poisson distribution to calculate the statistical $\chi_{\text {stat }}^{2}$,

$$
\chi_{\text {stat }}^{2}=\sum_{i} 2\left(N_{i}^{\text {test }}-N_{i}^{\text {true }}-N_{i}^{\text {true }} \log \frac{N_{i}^{\text {test }}}{N_{i}^{\text {true }}}\right) .
$$

Here, 'i' refers to the number of bins and $N_{i}^{\text {test }}, N_{i}^{\text {true }}$ are the total number of events due to test and true sets of oscillation parameters, respectively. $N_{i}^{\text {test }}$ is defined as follows, including the effect of systematics

$$
\begin{aligned}
N_{i}^{(\mathrm{k}) \text { test }}(\omega, \xi)= & \sum_{k=s, b} N_{i}^{(k)}(\omega)\left[1+c_{i}^{(k) \text { norm }} \xi^{(k) \text { norm }}\right. \\
& \left.+c_{i}^{(k) \text { tilt }} \xi^{(k) \text { tilt }} \frac{E_{i}-\bar{E}}{E_{\max }-E_{\min }}\right]
\end{aligned}
$$

where $k=s(b)$ represent the signal(background) events. $c_{i}^{\text {norm }}\left(c_{i}{ }^{\text {tilt }}\right)$ corresponds to the change in the number of 
events due to the pull variable $\xi^{\text {norm }}\left(\xi^{\text {tilt }}\right)$. In the above equation, $E_{i}$ denotes the mean reconstructed energy of the $i$ th bin with $E_{\min }$ and $E_{\max }$ representing the maximum and minimum energy in the entire energy range and $\bar{E}=$ $\left(E_{\max }+E_{\min }\right) / 2$ is the mean energy over this range. The systematic uncertainties (normalization errors) and efficiencies corresponding to signals and backgrounds of DUNE and HK are taken from [41,69]. For DUNE, the signal normalization uncertainties on $\nu_{e} / \overline{\nu_{e}}$ and $\nu_{\mu} / \overline{\nu_{\mu}}$ are considered to be $2 \%$ and $5 \%$ respectively. While a range of $5 \%$ to $20 \%$ background uncertainty along with the correlations among their sources have also been included. On the other hand, for T2HK the signal normalization error on $\nu_{e}\left(\overline{\nu_{e}}\right)$ and $\nu_{\mu}\left(\overline{\nu_{\mu}}\right)$ are considered to be $3.2 \%$ (3.9\%) and $3.6 \%(3.6 \%)$ respectively. In the case of T2HKK, 3.8\% $(4.1 \%)$ and $3.8 \%(3.8 \%)$ are taken as the signal normalization errors on $\nu_{e}\left(\overline{\nu_{e}}\right)$ and $\nu_{\mu}\left(\overline{\nu_{\mu}}\right)$ respectively. The background normalization uncertainties range from $3.8 \%$ to $5 \%$. $N_{i}{ }^{\text {true }}$ in Eq. (12) is obtained by adding the simulated signal and background events i.e., $N_{i}^{\text {true }}=N_{i}^{s}+N_{i}^{b}$.

In Table I, we list the values for the neutrino oscillation parameters that we have used in our numerical simulation. These values are consistent with the results obtained from the global fit of world neutrino data [24-26].

\section{A. Testing the $\sin ^{2} \theta_{23}-\delta_{C P}$ correlation predicted by the symmetries at DUNE, T2HK, and T2HKK}

The numerical analysis is performed as follows.

(i) The data corresponding to each experiment is generated by considering the true values of the oscillation parameters given in Table I. Note that the true values of $\theta_{23}$ and $\delta_{C P}$ are spanned over the range $\left(39-51^{\circ}\right)$ and $\left(0-360^{\circ}\right)$, respectively.

(ii) In the theoretical fit, we calculate the test events by marginalizing over the parameters $\sin ^{2} \theta_{13},\left|\Delta m_{31}^{2}\right|$, $\sin ^{2} \theta_{23}$ and $\sin ^{2} \theta_{12}$ in the test plane using the ranges presented in Table I.

(iii) The test values of $\delta_{C P}$ used are as predicted by the symmetries specified in Eq. (4) for $C_{1}$ and Eq. (5) in $C_{2}$.

TABLE I. Values of oscillation parameters that are considered in this study unless otherwise mentioned. We vary the true values of $\theta_{23}$ in the whole allowed range, and marginalization for each $\theta_{23}^{\text {true }}$ is done over the full allowed range of $\theta_{23}$. See text for more details.

\begin{tabular}{lcc}
\hline \hline Osc. param. & True values & Test values \\
\hline $\sin ^{2} \theta_{13}$ & 0.0219 & $0.0197-0.0244$ \\
$\sin ^{2} \theta_{12}$ & 0.306 & $0.272-0.346$ \\
$\theta_{23}$ & $39^{\circ}-51^{\circ}$ & $39^{\circ}-51^{\circ}$ \\
$\Delta m_{21}^{2}\left(\mathrm{eV}^{2}\right)$ & $7.50 \times 10^{-5}$ & Fixed \\
$\Delta m_{31}^{2}\left(\mathrm{eV}^{2}\right)$ & $2.50 \times 10^{-3}$ & $(2.35-2.65) \times 10^{-3}$ \\
$\delta_{C P}$ & $(0-360)^{\circ}$ & Symmetry predictions \\
\hline \hline
\end{tabular}

(iv) In addition we impose the conditions given in Eq. (8) for the symmetry relation $C_{1}$ and Eq. (9) for symmetry relation $C_{2}$ in the test. Note that given the current range of $\sin ^{2} \theta_{13}$ these relations restrict the value of $\sin ^{2} \theta_{12}$ to $0.316<\sin ^{2} \theta_{12}<0.319$ for $C_{1}$ and $\sin ^{2} \theta_{12}$ to $0.34<\sin ^{2} \theta_{12}<0.342$ for $C_{2}$. These values of $\sin ^{2} \theta_{12}$ are within the current $3 \sigma$ allowed range. Note that these ranges exclude the current best-fit value of $\sin ^{2} \theta_{12}$. Precise measurement of $\theta_{12}$, for instance, in the reactor neutrino experiment JUNO [75], can provide a stringent test of these scenarios.

(v) We have not added any prior in this analysis. We have checked that the prior on $\theta_{13}$ does not play any role when the constraints represented by Eqs. (8) and (9) are applied. In addition, since the best-fit $\theta_{12}$ is excluded already by the constraints, the imposition of $\theta_{12}$ prior will disfavor the scenarios.

(vi) We minimize the $\chi^{2}$ and plot the regions in the $\sin ^{2} \theta_{23}$ (true) $-\delta_{C P}($ true $)$ plane for which $\chi^{2} \leq \chi_{\min }^{2}+$ $\Delta \chi^{2}$ where $\Delta \chi^{2}$ values used correspond to $1 \sigma, 2 \sigma$, and $3 \sigma$.

The resultant plots are shown in Fig. 2 for true hierarchy as NH and Fig. 3 for true hierarchy as IH. We assume the hierarchy to be known, and we do not marginalize over hierarchy. ${ }^{3}$ The blue, gray, and yellow bands in Figs. 2 and 3 represent $1 \sigma, 2 \sigma, 3 \sigma$ regions in the $\sin ^{2} \theta_{23}-\delta_{C P}$ plane, respectively. The red contours show the $3 \sigma$ allowed area obtained by the Nu-fit Collaboration [26,63]. These plots show the extent to which these three experiments can test the correlations between the two yet undetermined variables $\sin ^{2} \theta_{23}$ and $\delta_{C P}$ in conjunction with the symmetry predictions. The red contours show the $3 \sigma$ allowed area obtained by the Nu-fit Collaboration [26,63]. The topmost panel corresponds to DUNE $40 \mathrm{kT}$ detector whereas the middle and the lowest panels correspond to T2HK and T2HKK experiments, respectively. The left plots in all the rows are for testing $C_{1}$ whereas the right plots are for testing $\mathrm{C}_{2}$.

The figures show that, because of the correlations predicted by symmetries, certain combinations of the true $\theta_{23}$ and $\delta_{C P}$ values get excluded by DUNE, T2HK and T2HKK. Owing to their high sensitivity to determine $C P$ violation, T2HK and T2HKK constrain the range of $\delta_{C P}$ better than that of DUNE. This can be seen from the figures (see Figs. 2,3) which show that, as we go from top to bottom the contours gets thinner with respect to $\delta_{C P}$. For instance, for the $C_{1}$ correlation, the $C P$ conserving values $0^{\circ}$ and $360^{\circ}$ get excluded at $3 \sigma$ for both of the octants by all three experiments, as can be seen from the plots in the left panels. However, for the $C_{2}$, these values are allowed at $3 \sigma$

\footnotetext{
${ }^{3}$ We verified that there is less of an effect of marginalizing over the hierarchy. Hence, to save the computation time, we have presented the plots by assuming that the hierarchy is known.
} 

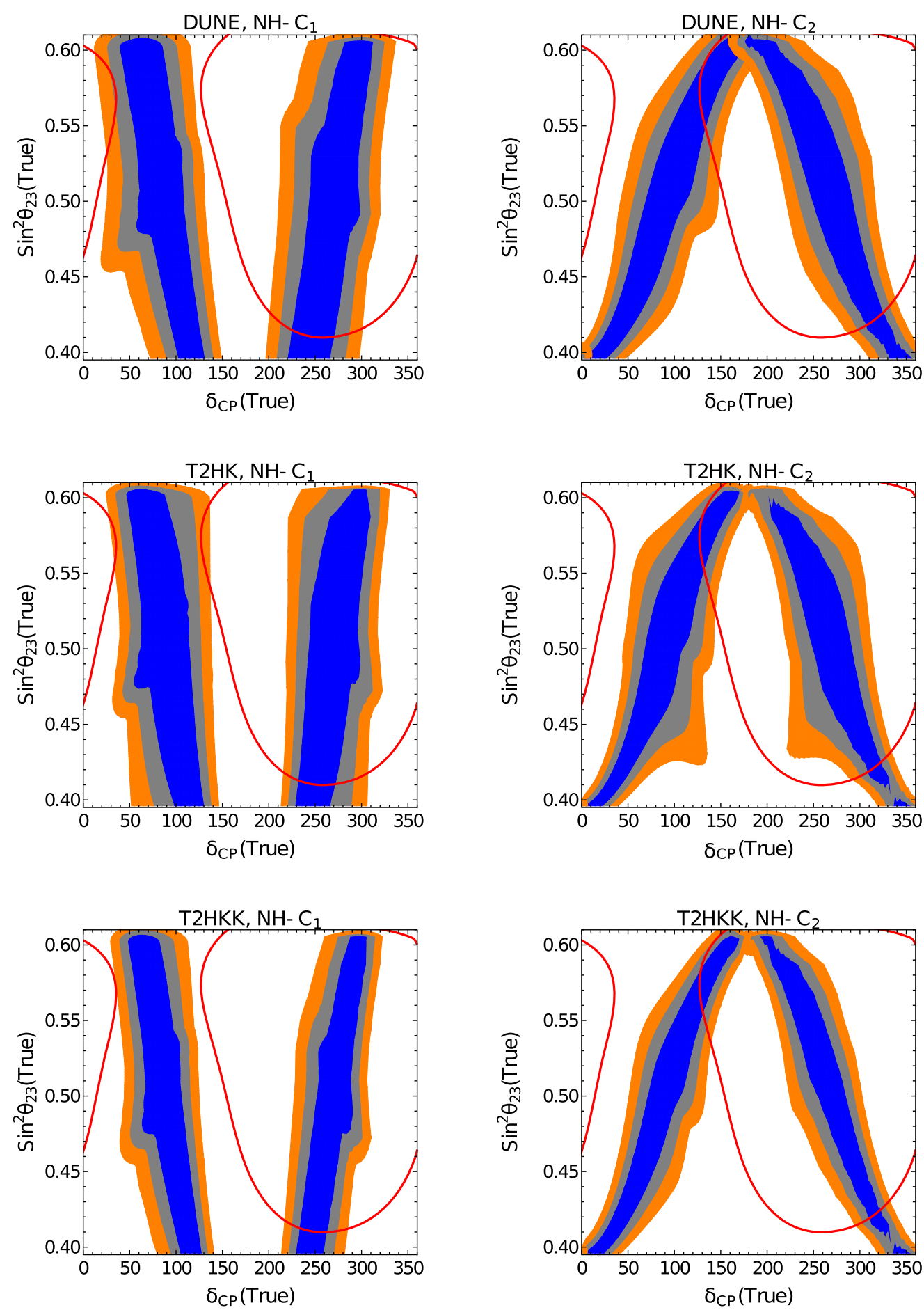

FIG. 2. Contour plots in the true: $\sin ^{2} \theta_{23}$ (true) $-\delta_{C P}$ (true) plane for DUNE, T2HK, and T2HKK. The left(right) panel represents the prediction from the symmetry relation $C_{1}\left(C_{2}\right)$ which corresponds to the Eq. (4) [Eq. (5)]. The hierarchy is fixed as NH. The red contour in each panel represents the $3 \sigma$ allowed area from the global analysis of neutrino oscillation data as obtained by the Nu-fit Collaboration $[26,63]$ for the normal hierarchy. The blue, gray, and yellow shaded contours correspond to $1 \sigma, 2 \sigma$, and $3 \sigma$ respectively.

for all three experiments. Whereas, $\delta_{C P}=0^{\circ}$ and $360^{\circ}$ are excluded by DUNE and HK experiments at $1 \sigma$ and $2 \sigma$, respectively. Again, one can see from the right panels that for $C_{2}, \delta_{C P}=180^{\circ}$ is allowed for $\sin ^{2} \theta_{23}>0.55$ (i.e., higher octant) by DUNE but barely gets excluded at $2 \sigma$ by the T2HK and T2HKK experiments. The correlations predicted by the symmetry considerations being independent of hierarchy, the allowed regions are not very different for $\mathrm{NH}$ and $\mathrm{IH}$, but the region of parameter space allowed by the current data for $\mathrm{IH}$ is more constrained and the 

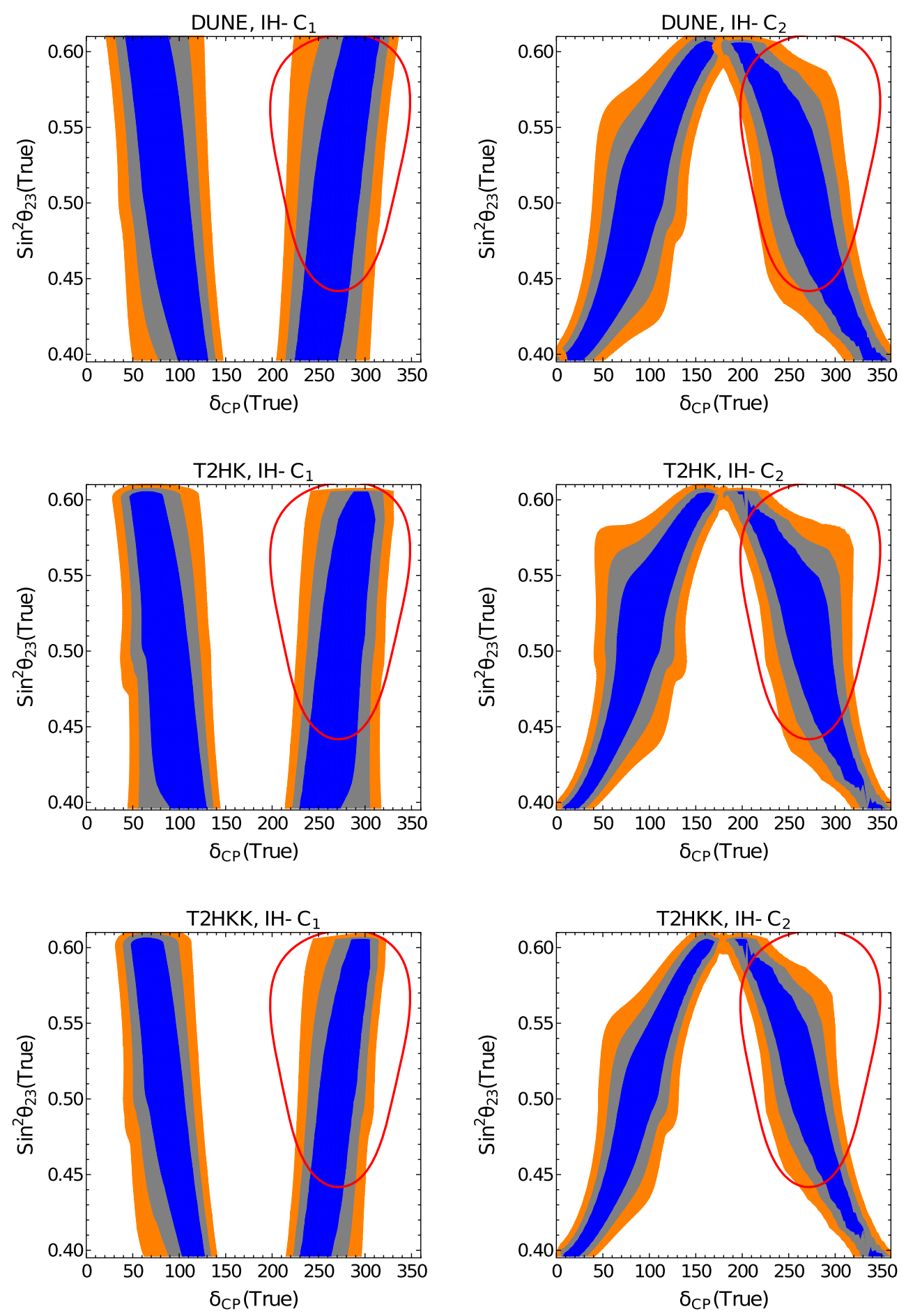

FIG. 3. Same as Fig. 2 but for inverted hierarchy.

symmetry predictions restrict it further, as can be seen from Fig. 3. Some of the parameter space allowed by the current data can also be disfavored by incorporating the correlations due to symmetry relations.

In Sec. II, it was discussed that the additional restrictions, Eqs. (8) and (9), are obtained when the partial $\mu$ - $\tau$ symmetry is derived in the specific approach discussed. However, possibilities exist where partial $\mu-\tau$ symmetry can be generated without the additional restrictions. In this context, we analyzed the changes in the allowed areas when the additional restrictions are not imposed. This is done only for DUNE, which captures the essential trend of the impact of not imposing the extra constraints. This is shown in Fig. 4. We have studied this for the representative case of the symmetry relation $C_{2}$. The procedure for generating the plots is the same as outlined earlier, except with the 

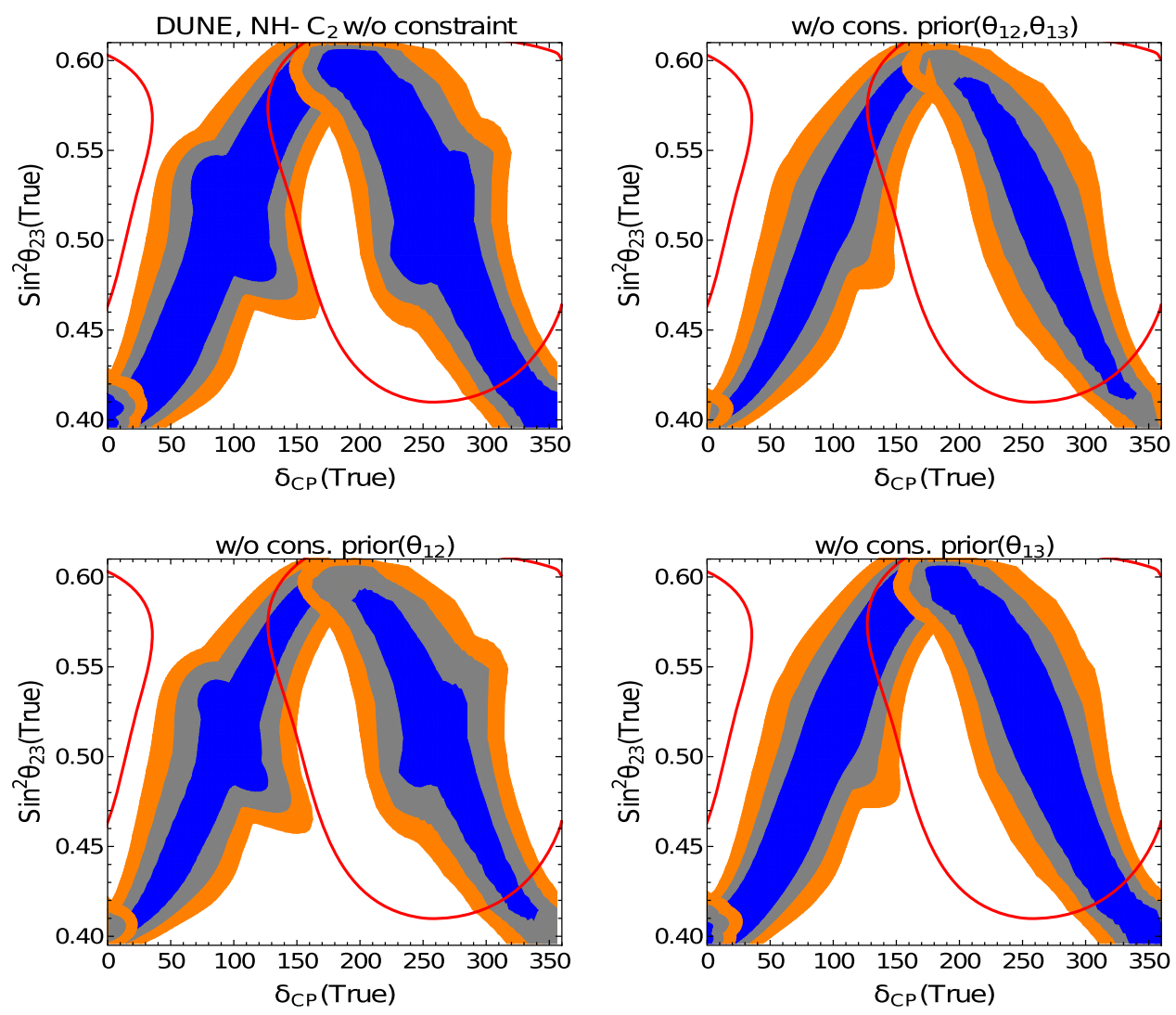

FIG. 4. Contour plots in the true: $\sin ^{2} \theta_{23}$ (true)- $\delta_{C P}$ (true) plane for DUNE, assuming the symmetry relation $C_{2}$ in the test. The additional constraints, Eq. (9), have not been applied in generating this plot. The first panel represents the plot without including any prior. The second panel in the first row shows the effect of the prior on $\theta_{12}$ and $\theta_{13}$. The first plot in the second row shows the effect of the inclusion of the $\theta_{12}$ prior, whereas the second plant shows the effect of the inclusion of the $\theta_{13}$ prior. The hierarchy is fixed as NH. The red contour in each panel represents the $3 \sigma$ allowed area of the Nu-fit Collaboration.

inclusion of the priors. While the earlier plots were generated without any prior, for these cases, we have studied the following scenarios:

(1) No prior on $\theta_{13}$ and $\theta_{12}$.

(2) Prior on $\theta_{13}$ and $\theta_{12}$.

(3) Prior on $\theta_{12}$ and no prior $\theta_{13}$.
(4) Prior on $\theta_{13}$ and no prior $\theta_{12}$.

The first plot of top row is without any prior on the parameters $\theta_{12}$ and $\theta_{13}$ and no additional constraints imposed. We find that the allowed area increases in size as compared to the cases where the extra constraints embodied in Eqs. (8) and (9) are not imposed. The second
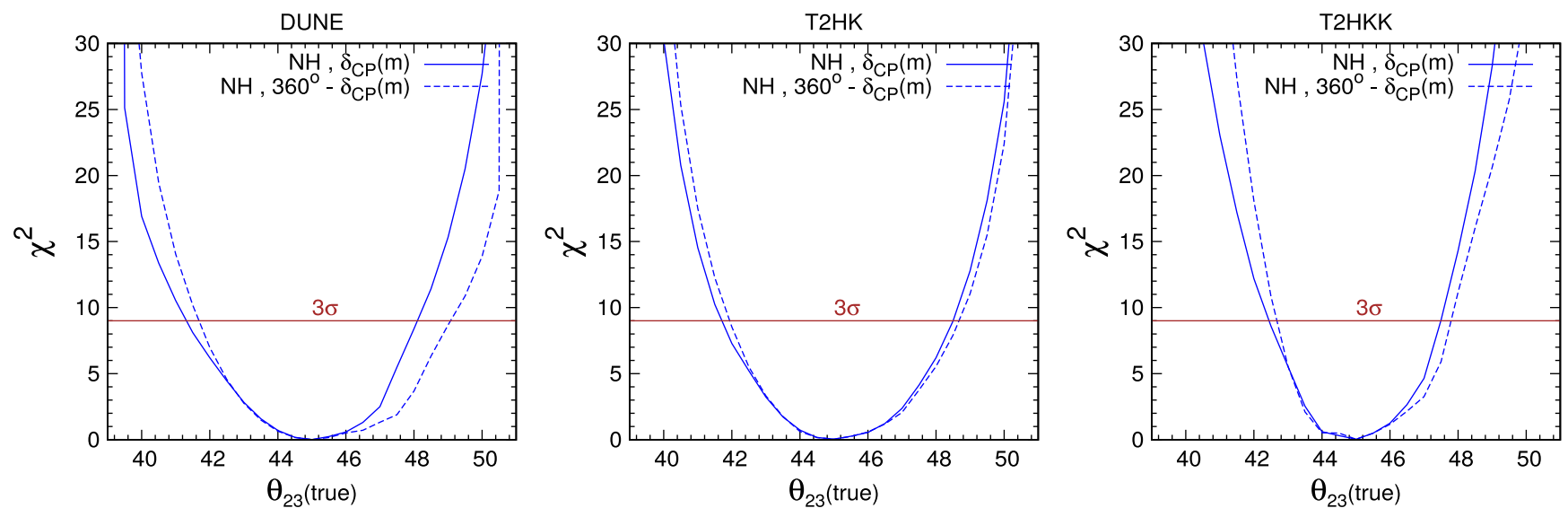

FIG. 5. The sensitivity of DUNE, T2HK, and T2HKK experiments to differentiate between $C_{1}$ and $C_{2}$ correlations for known normal hierarchy. 
TABLE II. The limits of $\theta_{23}$ in degrees below and above which the correlations $C_{1}$ and $C_{2}$ can be differentiated at $3 \sigma$ C.L. for two different ranges of $\delta_{C P}$.

\begin{tabular}{|c|c|c|c|c|c|c|}
\hline \multirow{2}{*}{$\begin{array}{l}\text { Range of } \delta_{C P} \\
0^{\circ} \leq \delta_{C P} \leq 180^{\circ} \\
180^{\circ} \leq \delta_{C P} \leq 360^{\circ}\end{array}$} & \multicolumn{2}{|c|}{ DUNE } & \multicolumn{2}{|c|}{ T2HK } & \multicolumn{2}{|c|}{ T2HKK } \\
\hline & $\begin{array}{l}\theta_{23} \leq 41.5^{\circ} \\
\theta_{23} \leq 41.8^{\circ}\end{array}$ & $\begin{array}{l}\theta_{23} \geq 48^{\circ} \\
\theta_{23} \geq 49^{\circ}\end{array}$ & $\begin{aligned} \theta_{23} & \leq 41.8^{\circ} \\
\theta_{23} & \leq 42^{\circ}\end{aligned}$ & $\begin{aligned} \theta_{23} & \geq 48.5^{\circ} \\
\theta_{23} & \geq 48.7^{\circ}\end{aligned}$ & $\begin{array}{l}\theta_{23} \leq 42.6^{\circ} \\
\theta_{23} \leq 42.8^{\circ}\end{array}$ & $\begin{array}{l}\theta_{23} \geq 47.5^{\circ} \\
\theta_{23} \geq 47.7^{\circ}\end{array}$ \\
\hline
\end{tabular}

plot of top row is without imposing these additional restrictions, but including the prior on $\theta_{12}$ and $\theta_{13}$. In this case, the allowed regions are more restricted and certain combinations of $\theta_{23}$ and $\delta_{C P}$ get disfavored. We proceed further to show the impact of the prior considering a single mixing angle at a time in the second row. In the first plot of the bottom row, we show the effect of including the prior on $\theta_{12}$ but no prior on $\theta_{13}$. In this case, the shape of the allowed regions are same but they reduce in size. The effect of certain combination of $\theta_{23}$ and $\delta_{C P}$ values getting disfavored are seen more at the $1 \sigma$ level. Similarly, the second plot of the bottom row shows the effect of the $\theta_{13}$ prior. In this case also, the allowed regions reduce in size as compared to the case where no priors are included (first panel of top row).

\section{B. Differentiating between the $C_{1}$ and $C_{2}$ symmetries}

In this subsection, we explore the possibility of differentiation between the symmetries $C_{1}$ and $C_{2}$. This is presented in Fig. 5, where we plot $\Delta \chi^{2}$ vs true $\theta_{23}$. To find $\chi_{\text {stat }}^{2}$ [as defined in Eq. (12)], true events are calculated by varying the true values of $\theta_{23}$ in the range $\left(39^{\circ}-51^{\circ}\right)$. For each true $\theta_{23}$, true values of $\sin ^{2} \theta_{13}$ and $\sin ^{2} \theta_{12}$ are allowed to vary in their $3 \sigma$ range such that the condition as given in Eq. (8) is satisfied. Using these true values of the angles the true $\delta_{C P}$ values are calculated using the correlation $C_{1}$. This leads to two sets of true events corresponding to $\delta_{C P}$ and $\left(360^{\circ}-\delta_{C P}\right)$, respectively. The remaining oscillation parameters are kept fixed at their best-fit values as shown in Table I. In the theoretical fit, to calculate test events, we marginalize over $\sin ^{2} \theta_{13},\left|\Delta m_{31}^{2}\right|, \sin ^{2} \theta_{23}$ in the range given in Table I, and test $\delta_{C P}$ values are calculated using the $C_{2}$. In addition, we impose the condition as given in Eq. (9), connecting test $\sin ^{2} \theta_{23}$ and $\sin ^{2} \theta_{13}$ and compute the $\chi^{2}$. For each choice of true $\sin ^{2} \theta_{23}$, the $\chi^{2}$ is marginalized over the true $\sin ^{2} \theta_{13}$ and $\sin ^{2} \theta_{12}$, and the minimum $\chi^{2}$ for each true $\sin ^{2} \theta_{23}$ is taken as the value of $\chi^{2}$. This process is done for both $\delta_{C P}$ and $360^{\circ}-\delta_{C P}$ separately. We have performed the analysis considering the true hierarchy as $\mathrm{NH}$. We have checked that if we assume the IH as the true hierarchy, we obtain similar results. The three panels from left to right represent DUNE, T2HK, and T2HKK, respectively. The solid blue curves in the plots are for the predicted range $\delta_{C P} \in\left(0^{\circ}<\delta_{C P}<180^{\circ}\right)$, and the dashed blue curves in the plots are for complementary range $360^{\circ}-\delta_{C P} \in\left(180^{\circ}<\delta_{C P}<360^{\circ}\right)$ as predicted by the correlations. The brown solid line shows the $3 \sigma$ C.L. We observe from the figure that at the maximal $\theta_{23}$, both of the correlations are indistinguishable by all the three experiments as is expected from Eqs. (4), (5).

The capability of the experiments to differentiate between the two correlations increases as we move away from the maximal value. The range of $\theta_{23}$, for which the three experiments can differentiate between the correlations at $3 \sigma$, is given in Table II. The lower limits signify the values of $\theta_{23}$ below which the correlations can be differentiated at $3 \sigma$, and the upper limits are for the values above which the same can be achieved.

\section{CONCLUSION}

We study here partial $\mu-\tau$ reflection symmetry of the leptonic mixing matrix, $U$, which can arise from discrete flavor symmetry. Specific assumptions which lead to this symmetry were reviewed here. This symmetry implies $\left|U_{\mu i}\right|=\left|U_{\tau i}\right|(i=1,2,3)$ for a single column of the leptonic mixing matrix $U$. If this is true for the third column of $U$, then it leads to maximal value of the atmospheric mixing angle and $C P$ phase $\delta_{C P}$. However, if this is true for the first or the second column, then one obtains definite correlations among $\theta_{23}$ and $\delta_{C P}$. We call these scenarios $C_{1}$ (equality for the first column) and $C_{2}$ (equality of the second column). We find that almost all the discrete subgroups of $\mathrm{SU}(3)$, except a few exceptional cases having three dimensional irreducible representations, display the form of partial $\mu-\tau$ symmetry. We study the correlations among $\theta_{23}$ and $\delta_{C P}$ in the two scenarios. Each scenario gives two values of $\delta_{C P}$ for a given $\theta_{23}$-one belonging to $0^{\circ}<\delta_{C P}<180^{\circ}$ and the other belonging to $180^{\circ}<\delta_{C P}<360^{\circ}$. The models also give specific correlations between $\theta_{23}$ and $\delta_{C P}$ and these are opposite for $C_{1}$ and $C_{2}$. We study how the allowed areas in the $\sin ^{2} \theta_{23}-\delta_{C P}$ plane obtained by the global analysis of neutrino oscillation data from the Nu-Fit Collaboration compare with the predictions from the symmetries.

We also expound the testability of these symmetries considering next generation accelerator based experiments, DUNE and Hyper-Kamiokande. This is illustrated in terms of plots in the $\sin ^{2} \theta_{23}$ (true) $-\delta_{C P}$ (true) plane obtained by fitting the simulated experimental data with the symmetry predictions for $\delta_{C P}$. The values of $\theta_{23}$ are found to be more constrained for the $C P$ conserving values namely $\delta_{C P}=0^{\circ}$, $180^{\circ}, 360^{\circ}$. For the $C_{2}$ correlation, the $\theta_{23}$ is found to be in the higher octant for $\delta_{C P}=180^{\circ}$ and in the lower octant for $\delta_{C P}=0^{\circ}$ and $360^{\circ}$. For the correlation $C_{1}$, values of $\delta_{C P}$ 
around all the three $C P$ conserving values $\delta_{C P}=0^{\circ}, 180^{\circ}$, and $360^{\circ}$ are seen to be disfavored. Finally, we illustrate the capability of DUNE and Hyper-Kamiokande to distinguish between the predictions of the two correlations. We observe that both the experiments can better differentiate between these two as one moves away from the maximal $\theta_{23}$ value.

In conclusion, the future experiments provide testing grounds for various symmetry relations, specially those connecting $\theta_{23}$ and $\delta_{C P}$.

\section{ACKNOWLEDGMENTS}

The research work of A. S. J. was supported by BRNS (Department of Atomic Energy) and by Department of Science and Technology, Government of India through the Raja Ramanna fellowship and the J.C. Bose Grant, respectively. N. N. gratefully acknowledges the support in part by the National Natural Science Foundation of China under Grant No. 11775231 for the research work.
[1] G. Altarelli and F. Feruglio, Rev. Mod. Phys. 82, 2701 (2010).

[2] G. Altarelli, F. Feruglio, and L. Merlo, Fortschr. Phys. 61, 507 (2013).

[3] A. Yu. Smirnov, J. Phys. Conf. Ser. 335, 012006 (2011).

[4] H. Ishimori, T. Kobayashi, H. Ohki, Y. Shimizu, H. Okada, and M. Tanimoto, Prog. Theor. Phys. Suppl. 183, 1 (2010).

[5] S. F. King and C. Luhn, Rep. Prog. Phys. 76, 056201 (2013).

[6] P. F. Harrison and W. G. Scott, Phys. Lett. B 547, 219 (2002).

[7] W. Grimus and L. Lavoura, Phys. Lett. B 579, 113 (2004).

[8] P. M. Ferreira, W. Grimus, L. Lavoura, and P. O. Ludl, J. High Energy Phys. 09 (2012) 128.

[9] W. Grimus and L. Lavoura, Fortschr. Phys. 61, 535 (2013).

[10] R. N. Mohapatra and C. C. Nishi, Phys. Rev. D 86, 073007 (2012).

[11] E. Ma, A. Natale, and O. Popov, Phys. Lett. B 746, 114 (2015).

[12] A. S. Joshipura and K. M. Patel, Phys. Lett. B 749, 159 (2015).

[13] A. S. Joshipura, J. High Energy Phys. 11 (2015) 186.

[14] A. S. Joshipura and N. Nath, Phys. Rev. D 94, 036008 (2016).

[15] C. C. Nishi and B. L. Snchez-Vega, J. High Energy Phys. 01 (2017) 068.

[16] Z.-h. Zhao, J. High Energy Phys. 09 (2017) 023.

[17] Z.-C. Liu, C.-X. Yue, and Z.-h. Zhao, J. High Energy Phys. 10 (2017) 102.

[18] Z.-z. Xing, D. Zhang, and J.-y. Zhu, J. High Energy Phys. 11 (2017) 135.

[19] Z.-z. Xing and J.-y. Zhu, Chin. Phys. C 41, 123103 (2017).

[20] A. S. Joshipura, arXiv:1801.02843.

[21] N. Nath, Z.-Z. Xing, and J. Zhang, Eur. Phys. J. C 78, 289 (2018).

[22] Z.-h. Zhao, Nucl. Phys. B935, 129 (2018).

[23] C. Patrignani et al. (Particle Data Group), Chin. Phys. C 40, 100001 (2016).

[24] P. F. de Salas, D. V. Forero, C. A. Ternes, M. Tortola, and J. W. F. Valle, Phys. Lett. B 782, 633 (2018).

[25] F. Capozzi, E. Lisi, A. Marrone, D. Montanino, and A. Palazzo, Nucl. Phys. B908, 218 (2016).

[26] I. Esteban, M. C. Gonzalez-Garcia, M. Maltoni, I. MartinezSoler, and T. Schwetz, J. High Energy Phys. 01 (2017) 087.
[27] T. Fukuyama and H. Nishiura, arXiv:hep-ph/9702253.

[28] E. Ma and M. Raidal, Phys. Rev. Lett. 87, 011802 (2001); 87, 159901(E) (2001).

[29] C. S. Lam, Phys. Lett. B 507, 214 (2001).

[30] K. R. S. Balaji, W. Grimus, and T. Schwetz, Phys. Lett. B 508, 301 (2001).

[31] W. Grimus, A. S. Joshipura, S. Kaneko, L. Lavoura, H. Sawanaka, and M. Tanimoto, Nucl. Phys. B713, 151 (2005).

[32] A. S. Joshipura, Eur. Phys. J. C 53, 77 (2008).

[33] Z.-z. Xing and Z.-h. Zhao, Rep. Prog. Phys. 79, 076201 (2016).

[34] Z.-z. Xing and S. Zhou, Phys. Lett. B 737, 196 (2014).

[35] S.-F. Ge, D. A. Dicus, and W. W. Repko, Phys. Lett. B 702, 220 (2011).

[36] S.-F. Ge, D. A. Dicus, and W. W. Repko, Phys. Rev. Lett. 108, 041801 (2012).

[37] S. T. Petcov, Eur. Phys. J. C 78, 709 (2018).

[38] R. de Adelhart Toorop, F. Feruglio, and C. Hagedorn, Phys. Lett. B 703, 447 (2011).

[39] A. D. Hanlon, S.-F. Ge, and W. W. Repko, Phys. Lett. B 729, 185 (2014).

[40] A. D. Hanlon, W. W. Repko, and D. A. Dicus, Adv. High Energy Phys. 2014, 469572 (2014).

[41] R. Acciarri et al. (DUNE Collaboration), arXiv:1512.06148.

[42] V. Barger, A. Bhattacharya, A. Chatterjee, R. Gandhi, D. Marfatia, and M. Masud, Phys. Rev. D 89, 011302 (2014).

[43] S. K. Agarwalla, S. Prakash, and S. Uma Sankar, J. High Energy Phys. 03 (2014) 087.

[44] V. Barger, A. Bhattacharya, A. Chatterjee, R. Gandhi, D. Marfatia, and M. Masud, Int. J. Mod. Phys. A 31, 1650020 (2016).

[45] K. Bora, D. Dutta, and P. Ghoshal, Mod. Phys. Lett. A 30, 1550066 (2015).

[46] M. Ghosh, S. Goswami, and S. K. Raut, Eur. Phys. J. C 76, 114 (2016).

[47] D. Dutta and k. Bora, Mod. Phys. Lett. A 30, 1550017 (2015).

[48] K. N. Deepthi, C. Soumya, and R. Mohanta, New J. Phys. 17, 023035 (2015).

[49] N. Nath, M. Ghosh, and S. Goswami, Nucl. Phys. B913, 381 (2016).

[50] R. Srivastava, C. A. Ternes, M. Trtola, and J. W. F. Valle, Phys. Rev. D 97, 095025 (2018).

[51] P. Pasquini, Adv. High Energy Phys. 2018, 1825874 (2018). 
[52] R. Srivastava, C. A. Ternes, M. Trtola, and J. W. F. Valle, Phys. Lett. B 778, 459 (2018).

[53] S. K. Agarwalla, S. S. Chatterjee, S. T. Petcov, and A. V. Titov, Eur. Phys. J. C 78, 286 (2018).

[54] S. S. Chatterjee, M. Masud, P. Pasquini, and J. W. F. Valle, Phys. Lett. B 774, 179 (2017).

[55] S. T. Petcov and A. V. Titov, Phys. Rev. D 97, 115045 (2018).

[56] P. Ballett, S. F. King, S. Pascoli, N. W. Prouse, and T. Wang, J. High Energy Phys. 03 (2017) 110.

[57] P. Ballett, S. Pascoli, and J. Turner, Phys. Rev. D 92, 093008 (2015).

[58] P. Ballett, S. F. King, C. Luhn, S. Pascoli, and M. A. Schmidt, J. High Energy Phys. 12 (2014) 122.

[59] J. T. Penedo, S. T. Petcov, and T. Yanagida, Nucl. Phys. B929, 377 (2018).

[60] S. F. King and T. Neder, Phys. Lett. B 736, 308 (2014).

[61] A. S. Joshipura and K. M. Patel, J. High Energy Phys. 01 (2017) 134.

[62] W. Grimus and P. O. Ludl, J. Phys. A 47, 075202 (2014).

[63] NuFIT, NuFIT 3.2, http://www.nu-fit.org/.
[64] P. Huber, M. Lindner, and W. Winter, Comput. Phys. Commun. 167, 195 (2005).

[65] P. Huber, J. Kopp, M. Lindner, M. Rolinec, and W. Winter, Comput. Phys. Commun. 177, 432 (2007).

[66] M. D. Messier, Ph.D. thesis, Boston University, 1999.

[67] E. Paschos and J. Yu, Phys. Rev. D 65, 033002 (2002).

[68] T. Alion et al. (DUNE Collaboration), arXiv:1606.09550.

[69] K. Abe et al. (hyper-Kamiokande Proto-Collaboration), Prog. Theor. Exp. Phys. 2018, 063C01 (2018).

[70] C. Adams et al. (LBNE Collaboration), arXiv:1307.7335.

[71] D. Cherdack, Private communication (2014).

[72] M. C. Gonzalez-Garcia and M. Maltoni, Phys. Rev. D 70, 033010 (2004).

[73] G. Fogli, E. Lisi, A. Marrone, D. Montanino, and A. Palazzo, Phys. Rev. D 66, 053010 (2002).

[74] R. Gandhi, P. Ghoshal, S. Goswami, P. Mehta, S. Uma Sankar, and S. Shalgar, Phys. Rev. D 76, 073012 (2007).

[75] Y.-F. Li (JUNO Collaboration), in Proceedings, 17th Lomonosov Conference on Elementary Particle Physics: Moscow, Russia, 2015 (2017), pp. 27-33 [arXiv:1606 .04743], https://inspirehep.net/record/1469449/files/arXiv: 1606.04743.pdf. 